This is an electronic reprint of the original article. This reprint may differ from the original in pagination and typographic detail.

\author{
Author(s): Rantakokko, Merja; Portegijs, Erja; Viljanen, Anne; Iwarsson, Susanne; Rantanen, \\ Taina
}

Title: Life-space mobility and quality of life in community-dwelling older people

Year: $\quad 2013$

Version:

Please cite the original version:

Rantakokko, M., Portegijs, E., Viljanen, A., Iwarsson, S., \& Rantanen, T. (2013). Lifespace mobility and quality of life in community-dwelling older people. Journal of the American Geriatrics Society, 61(10), 1830-1832. https://doi.org/10.1111/jgs.12473

All material supplied via JYX is protected by copyright and other intellectual property rights, and duplication or sale of all or part of any of the repository collections is not permitted, except that material may be duplicated by you for your research use or educational purposes in electronic or print form. You must obtain permission for any other use. Electronic or print copies may not be offered, whether for sale or otherwise to anyone who is not an authorised user. 


\section{Life-space mobility and quality of life among community-dwelling older people}

\section{Merja Rantakokko ${ }^{1}, \mathrm{PhD}$, Erja Portegijs ${ }^{1}, \mathrm{PhD}$, Anne Viljanen ${ }^{1}, \mathrm{PhD}$,} Susanne Iwarsson ${ }^{2}, \mathrm{PhD}$ and Taina Rantanen ${ }^{1}, \mathrm{PhD}$.

${ }^{1}$ Gerontology Research Center and Department of Health Sciences, University of Jyväskylä, Finland

${ }^{2}$ Department of Health Sciences, Lund University, Sweden

Corresponding author:

Merja Rantakokko

Gerontology Research Center and Department of Health Sciences

P.O. Box 35

FI-40014 University of Jyväskylä, Finland

tel. +358408053589

E-mail: Merja.rantakokko@jyu.fi

Alternate corresponding author:

Erja Portegijs

Gerontology Research Center and Department of Health Sciences

P.O. Box 35

FI-40014 University of Jyväskylä, Finland

e-mail: erja.portegijs@jyu.fi

Funding and related paper presentations

"Life-space mobility in old age" (LISPE) project is funded by the Academy of Finland, the Future of Living and Housing (ASU-LIVE; grant number 255403) program and the Finnish 
Ministry of Education and Culture. Additionally Anne Viljanen is funded by the Academy of Finland (grant numbers 251723, 263729). The funding agencies played no role in the design, conduct, data management, analysis or manuscript preparation related to this article.

The abstract of this paper is submitted to be presented in the $66^{\text {th }}$ Annual Scientific Meeting of the Gerontological Society of America, New Orleans, 20.-24.11.2013.

Running head: Life-space mobility and quality of life 


\section{LETTER TO THE EDITOR \\ RESEARCH}

\section{Life-space mobility and quality of life among community-dwelling older people}

To the Editor: Ability to go where and when a person wants to go is a key determinant of wellbeing in old age. ${ }^{1}$ The concept of life-space mobility ${ }^{2}$ reflects how much people actually move in the community and exploit community amenities as well as maintain social relationships and roles, and participate in meaningful activities. Life-space mobility correlates e.g. with age, gender, physical functioning, depressive symptoms, ${ }^{2,3}$ frailty and mortality, ${ }^{4}$ and the physical and mental components of quality of life (QoL). ${ }^{2}$ However, QoL is a multidimensional concept which reflects the total wellbeing of a person, and thus also includes social and environmental components. Few studies have focused on life-space mobility and overall QoL among older community-dwelling people, and we are unaware of studies on the association between life-space mobility and the social and environmental domains of QoL. The aim of this study was to examine the association between life-space mobility and different domains of QoL among community-dwelling people.

This paper is based on the baseline data of a project entitled "Life-space mobility in old age" (LISPE). The study design and methods have been reported in detail elsewhere. ${ }^{5}$ A total of 848, community-dwelling, 75- to 90-year-old people (mean age 80.1 years, $62 \%$ women) were interviewed in their homes. The Ethical Committee of the University of Jyväskylä, Finland, approved the project and all participants signed a written informed consent.

QoL was assessed with the World Health Organization QoL Assessment short version, WHOQOL-BREF. ${ }^{6}$ Scores were calculated separately for physical, psychological, social, and 
environmental domains. The total QoL score comprising all domains ranges from 0 to 130; higher scores indicate better QoL. ${ }^{6}$

Life-space mobility was measured with the Life-Space Assessment (LSA). ${ }^{2,5}$ Four indicators of life-space mobility were calculated: 1) maximal life-space (LSA-M), indicating the greatest distance attained with the help of assistive devices and/or another person if needed; 2) independent life-space (LSA-I), indicating the life-space attained without help from any assistive devices or another person; 3) life-space using assistive devices (LSA-A), indicating the life-space attained using the help of assistive devices if needed but not the help of another person; and 4) a composite score (LSA-C) reflecting distance, frequency and independency of movement (range 0-120); higher scores indicate larger life-space. ${ }^{2}$

Complete QoL data were available for 845 participants. The mean total QoL score was 100.3 ( \pm SD 11.8). The mean LSA-C score was $64.0( \pm 20.6)$. The LSA-C score correlated with all domains of QoL with the strongest correlation observed for the total QoL score (Table 1.) Linear regression analysis was used to assess the strength of the association between lifespace indicators and the total QoL score. When adjusted for age and sex, the standardized $\beta$ was $0.467(\mathrm{p}<.001)$ for LSA-C and the total QoL score. The model explained $23 \%$ of the variation in QoL. For LSA-M, LSA-I and LSA-A the corresponding values were $\beta=.172$; $\beta=.393, \beta=.281$, for all associations $p>.001$. Further adjustment for education, perceived financial position, number of self-reported physician-diagnosed chronic conditions and cognitive impairment slightly reduced the $\beta$-coefficients, but all associations remained statistically significant $(\mathrm{p}<.001)$.

Life-space mobility is associated with all QoL domains and overall QoL. Since a life-space assessment ${ }^{2}$ also takes into account the environment in which a person moves, it 
is understandable that an association was observed between the environmental QoL and lifespace mobility. Environmental factors may support or restrict the ability to move outdoors ${ }^{7}$ and to take part in social activities. ${ }^{8}$ However, the association between life-space mobility and social QoL was rather weak. It is possible that satisfaction with social life and social contacts are not fully dependent on ability to meet people in person, ${ }^{9}$ and presumably, social interaction does also take place within the home. Thus, the size of life-space is not likely to have considerable effect on social QoL.

Of the life-space indicators, LSA-M had the weakest association with overall QoL. Since the measurement of LSA-M does not take into account how or why participants have attained their life-space level, it is possible a participant has been taken by another person, for example to see a doctor. This appears as a larger life-space, despite the fact that the participant has not actively travelled outside home. An increase in life-space of this kind is not likely to improve QoL. It is important that the individual is satisfied with the level and quality of participation. ${ }^{10}$

In conclusion, larger life-space and good QoL coincide among older people, regardless of their health situation. Guaranteeing the possibility to go where and when one wants may promote QoL among older people. 


\section{ACKNOWLEDGMENTS}

We are grateful to Eeva-Maija Palonen, MSc, for her valuable work as a coordinator of the LISPE project and Marja-Liisa Kinnunen, $\mathrm{MD}, \mathrm{PhD}$, for her contribution to the coding of chronic diseases. We thank all study participants and interviewers for their contribution. The Gerontology Research Center (GEREC) is a joint effort between the universities of Jyväskylä and Tampere, Finland.

\section{Author Contributions}

The authors are justifiably credited with authorship, according to the authorship criteria. MR: conception, design, data collection, analysis and interpretation of the data, writing the article; EP: conception, design, data collection, critical revision of the article; AV: conception, design, data collection, critical revision of the article. SI: conception, design, critical revision of the article; TR: conception, design, data collection, critical revision of the article, PI for the LISPE project. All the authors approved the final manuscript.

Sponsor's Role: None. 


\section{REFERENCES}

1. Satariano WA, Guralnik JM, Jackson RJ, Marottoli RA, Phelan EA, Prohaska TR. Mobility and aging: New directions for public health action. Am J Public Health. 2012;102:1508-1515.

2. Baker PS, Bodner EV, Allman RM. Measuring life-space mobility in community-dwelling older adults. J Am Geriatr Soc. 2003;51:1610-1614.

3. Al Snih S, Peek KM, Sawyer P, Markides KS, Allman RM, Ottenbacher KJ. Life-space mobility in Mexican Americans aged 75 and older. J Am Geriatr Soc. 2012;60:532-537.

4. Xue QL, Fried LP, Glass TA, Laffan A, Chaves PH. Life-space constriction, development of frailty, and the competing risk of mortality: The women's health and aging study I. Am J Epidemiol. 2008;167:240-248.

5. Rantanen T, Portegijs E, Viljanen A, et al. Individual and environmental factors underlying life space of older people - study protocol and design of a cohort study on life-space mobility in old age (LISPE). BMC Public Health. 2012;12:1018. doi: 10.1186/1471-2458-12-1018.

6. The WHOQOL Group. Development of the world health organization WHOQOL-BREF quality of life assessment. Psychol Med. 1998;28:551-558.

7. Rantakokko M, Iwarsson S, Mänty M, Leinonen R, Rantanen T. Perceived barriers in the outdoor environment and development of walking difficulties in older people. Age Ageing. $2012 ; 41: 118-121$. 
8. Richard L, Gauvin L, Gosselin C, Laforest S. Staying connected: Neighbourhood correlates of social participation among older adults living in an urban environment in Montreal, Quebec. Health Promot Int. 2009;24:46-57.

9. Pinquart M, Sorensen S. Influences of socioeconomic status, social network, and competence on subjective well-being in later life: A meta-analysis. Psychol Aging. 2000;15:187-224.

10. Levasseur M, Desrosiers J, St-Cyr Tribble D. Subjective quality-of-life predictors for older adults with physical disabilities. Am J Phys Med Rehabil. 2008;87:830-841. 
Table 1. Spearman Correlation Coefficients between Quality of Life and Life-Space Mobility among Community-Dwelling Older People, N=845.

\begin{tabular}{llllll}
\hline LSA & QoL & \multicolumn{5}{c}{ QoL domain } \\
\cline { 3 - 6 } indicator & total score & Physical & Psychological & Social & Environmental \\
& & & & & \\
\hline LSA-C & $.464 * *$ & $.490^{* *}$ & $.279 * *$ & $.100^{* *}$ & $.373^{* *}$ \\
LSA-M & $.220^{* *}$ & $.238^{* *}$ & $.111^{* *}$ & .014 & $.181^{* *}$ \\
LSA-I & $.393^{* *}$ & $.449^{* *}$ & $.198^{* *}$ & .038 & $.311^{* *}$ \\
LSA-A & $.308^{* *}$ & $.346^{* *}$ & $.162^{* *}$ & -.003 & $.250^{* *}$ \\
\hline
\end{tabular}

$* * \mathrm{p}<.001$

QoL $=$ Quality of Life ${ }^{6}$

LSA $=$ Life-Space Assessment ${ }^{2}$

LSA-C $=$ Life-Space Assessment, Composite score

LSA-M= Life-Space Assessment, Maximal life-space score

LSA-I $=$ Life-Space Assessment, Independent life-space score

LSA-A= Life-Space Assessment, Assisted with mobility device life-space score 